\title{
Consideraciones del Leiomioma y su Variante Intraósea en Mandíbula, Algoritmo de Trabajo Según Análisis de Literatura
}

\author{
Considerations of Leiomyoma and its Intraosseous Variant in \\ the Jaw, Working Algorithm According to Literature Analysis
}

Daniel Pino Díaz; Claudio Huentequeo-Molina ${ }^{1,2,4}$; Alejandro Unibazo ${ }^{1,4}$; Saul Siso ${ }^{1}$ \& Sergio Olate ${ }^{1,2,3}$

PINO, D. D.; HUENTEQUEO-MOLINA, C.;UNIBAZO, A.; SISO, S. \& OLATE, S. Consideraciones del Leiomioma y su variante intraósea en mandíbula, algoritmo de trabajo según análisis de literatura. Int. J. Odontostomat., 14(1):117-123, 2020.

RESUMEN: EI Leiomioma es una neoplasia benigna originada en el tejido muscular liso por lo que puede manifestarse en cualquier región del cuerpo humano que contenga músculo liso, siendo el sitio más común el útero, la piel y en el tracto gastrointestinal. La escasa cantidad de tejido muscular liso en boca hace que su manifestación oral sea infrecuente, representando solo $0.06 \%$ del total de este tipo de tumores. Los sitios más afectados suelen ser los labios, las mejillas, el paladar, la lengua y encías. Histológicamente se distinguen tres tipos: el Leiomioma Sólido, Angioleiomioma (Vascular) y Leiomioma Epitelioide (Leiomioblastoma). El origen de esta neoplasia en boca suele ser la túnica media de los vasos sanguíneos. El Leiomioma intraóseo suele ser aún menos frecuente, y con un diagnóstico diferencial complejo, con histopatología que en varias ocasiones no suele ser fácil de clasificar. Si bien es definida como un tumor benigno, su manifestación intraósea puede llegar a ser localmente agresiva y con un diagnóstico controversial, debiendo abordarse muchas veces como una neoplasia maligna. El objetivo de este artículo es presentar una revisión de la literatura de esta variante intraósea de Leiomioma situada en mandíbula, sus consideraciones clínicas y un algoritmo de tratamiento.

PALABRAS CLAVE: cavidad oral, mandíbula, mandíbula, leiomioma, angiomioma, leiomioma epitelioide.

\section{INTRODUCCIÓN}

El Leiomioma es un tumor benigno originado en el tejido muscular liso, por lo que su aparición puede situarse en cualquier región del cuerpo humano que posea músculo liso. De esta forma existe un predominio en el útero, la piel y el tracto gastrointestinal (Eichner, 1963). El primer caso fue reportado por Blanc en 1884 y desde entonces se piensa que menos del 1 $\%$ se ubicaría en la región de cabeza y cuello, y solo un $0,06 \%$ se encontraría en la cavidad oral (Baden et al., 1994). Considerando todos los tumores benignos de la cavidad oral, el leiomioma corresponde aproximadamente el 0,42 \% (Brooks et al., 2002), y probablemente el sitio de origen de esta neoplasia sea la túnica media de vasos sanguíneos y células musculares lisas de conductos excretores de glándulas salivares (Gianluca et al).
El leiomioma fue definido por la Organización Mundial de la Salud (OMS) en 2002 (Brooks et al.), como un tumoración circunscrita benigna, a veces cutánea, compuesto por manojos de células musculares lisas entrecruzadas. Histológicamente se distinguen tres tipos: angioleiomioma o vascular $(74 \%$ de los casos), leiomioma sólido (25\%), y leiomioma epitelioide o leiomioblastoma, con un solo caso descrito en la literatura (Leung et al., 1990). El angioleiomioma es la versión más frecuente en la cavidad oral atribuido principalmente al alto contenido vascular de la región (Gaitan Cepeda et al., 2008).

Clínicamente el leiomioma oral se caracteriza por una masa nodular solida menor a $2 \mathrm{~cm}$, de crecimiento lento, localizada principalmente en tejidos

\footnotetext{
${ }^{1}$ Unidad de Cirugía Oral y Maxilofacial e Implantología, Hospital Dr. Abraham Godoy Peña, Lautaro de Chile.

2 División de Cirugía Oral y Maxilofacial, Universidad de Los Andes, Chile.

${ }^{3}$ Centro de Investigación Biomédica, Universidad de La Frontera, Chile.

${ }^{4}$ División de Cirugía Oral y Maxilofacial, Universidad de La Frontera, Chile.
} 
gingivales, lengua, labios, paladar, glándulas salivales y mucosa yugal (Savage et al., 1983; Schenberg et al., 1993). Su ocurrencia en tejidos duros (intraóseo), como la mandíbula, suele ser infrecuente (McMillan et al., 1986; Raffaini et al., 1990; Loyola et al., 1999). Generalmente suele ser una lesión asintomática, sin embargo síntomas como dolor, movilidad dentaria o dificultad al masticar pueden estar presentes. (Gianluca)

Debido a su inespecífica presentación clínica , es muy difícil diferenciar el leiomioma mandibular intraóseo de otra neoplasia mesenquimática como el fibroma, neurofibroma, lipoma o su contraparte el leiomiosarcoma o el miofibrosarcoma. por lo que para su diagnóstico,el análisis histológico y ultraestructural parecen ser fundamentales. Los diagnósticos diferenciales incluirían aquellas patologías de glándulas salivales (mucocele, adenoma pleomórfico), tumores vasculares (linfangioma, hemangioma, granuloma piogénico), y quistes de tejidos blando como quistes dermoides. El objetivo de este artículo es presentar una revisión de literatura y actualización en los conocimientos sobre el leiomioma intraóseo en mandíbula. Presentando un algoritmo de tratamiento y las especiales consideraciones que se deben tener cuando esta patología se origina dentro de tejido óseo.

Manifestaciones clínicas y radiográficas. La literatura consigna que la variante más frecuente de Leiomioma es el angioleiomioma seguido del leiomioma sólido. La manifestación clínica es una masa irregular de tejidos blando que por lo general corresponde a un aumento de volumen asintomático (9 casos), pero podría llegar a ser dolorosa (4 casos), 4 pacientes no presentaron ninguna manifestación clínica (hallazgo radiográfico), y uno relató una sensación de disconfort (Tabla I).

En nuestra revisión encontramos 20 casos que afectaron la mandíbula y 4 el maxilar. Catorce casos comprometieron la región posterior de la mandíbula (en 2 no especifica), y 4 comprometieron afectaron tanto la región posterior como anterior de este hueso. En hueso maxilar, un caso afectó al paladar, dos casos la región posterior, y uno la región anterior y posterior.

La afección de las tablas corticales se distribuyó con 9 casos donde se observó perforación, en 3 se observó expansión de estas, en 1 una discreta reabsorción horizontal del proceso alveolar, y en dos no se pesquisaron alteraciones.
El leiomioma clínicamente se distingue por ser un aumento de volumen, que por lo general son $>2 \mathrm{~cm}$, indoloros y de crecimiento lento. Sin embargo cuando su origen es intraóseo (mandíbula), posiblemente derivado del músculo liso vascular, suele presentar un crecimiento rápido y agresivo, con movilidad de piezas dentarias, abombamiento de corticales óseas y sangramiento.

La imagen radiográfica del leiomioma intraóseo, se presenta como una lesión radiolúcida, la cual puede ser uni o multilocular (Fig. 1). Concordando así con la literatura, radiográficamente el hallazgo más frecuente en nuestra revisión fueron lesiones radiolúcidas $(20$ casos), de las cuales 3 se presentaron apariencia multilocular y en 4 se distinguieron márgenes escleróticos. La rizálisis de piezas adyacentes se identificaron en 4 casos. En dos casos la lesión compromete territorios vecinos. Solo en un caso se observa un velamiento completo del seno maxilar. El tamaño de la lesión fue por lo general mayor o igual a $2 \mathrm{~cm}$ en promedio (14 casos). Cabe destacar que de todos los casos encontrados, 4 corresponden a hallazgos radiográficos.

Histología. Según la OMS, el Leiomioma se divide en tres tipos histológicos: el angioleiomioma, el leiomioma epitelioide y leiomioblastoma (Gianluca et al.). La Histopatología del Leiomioma se compone de una proliferación circunscrita, no encapsulada, de células musculares lisas en fascículos de barrido; así en los angioleiomiomas o leiomiomas vasculares, los fascículos pueden mostrar una configuración espiral alrededor de un espacio central revestido de endotelio; el leiomioma epitelioide se compone principalmente de células epitelioides en lugar de células fusiformes (Loyola et al.; Chaudhry et al.).

Las células musculares lisas poseen un citoplasma pálido, eosinofílico, vacuolado, y núcleos alargados, a veces en "forma de cigarro (extremos romos)". Sin evidencia de atipía citológica, mientras las mitóticas son infrecuentes. Por otro lado el músculo liso se tiñe de rojo brillante con tricrómico de Masson. Otra de las características es que el citoplasma es positivo para vimentina, actina del músculo liso, actina músculo específica, además se puede apreciar la positividad de desmina.

En cuanto al diagnóstico diferencial histopatológico del Leiomioma debemos reconocer varios diagnósticos como ya mencionamos. Por su parte el miofibroma en contraste con el leiomioma, tie- 


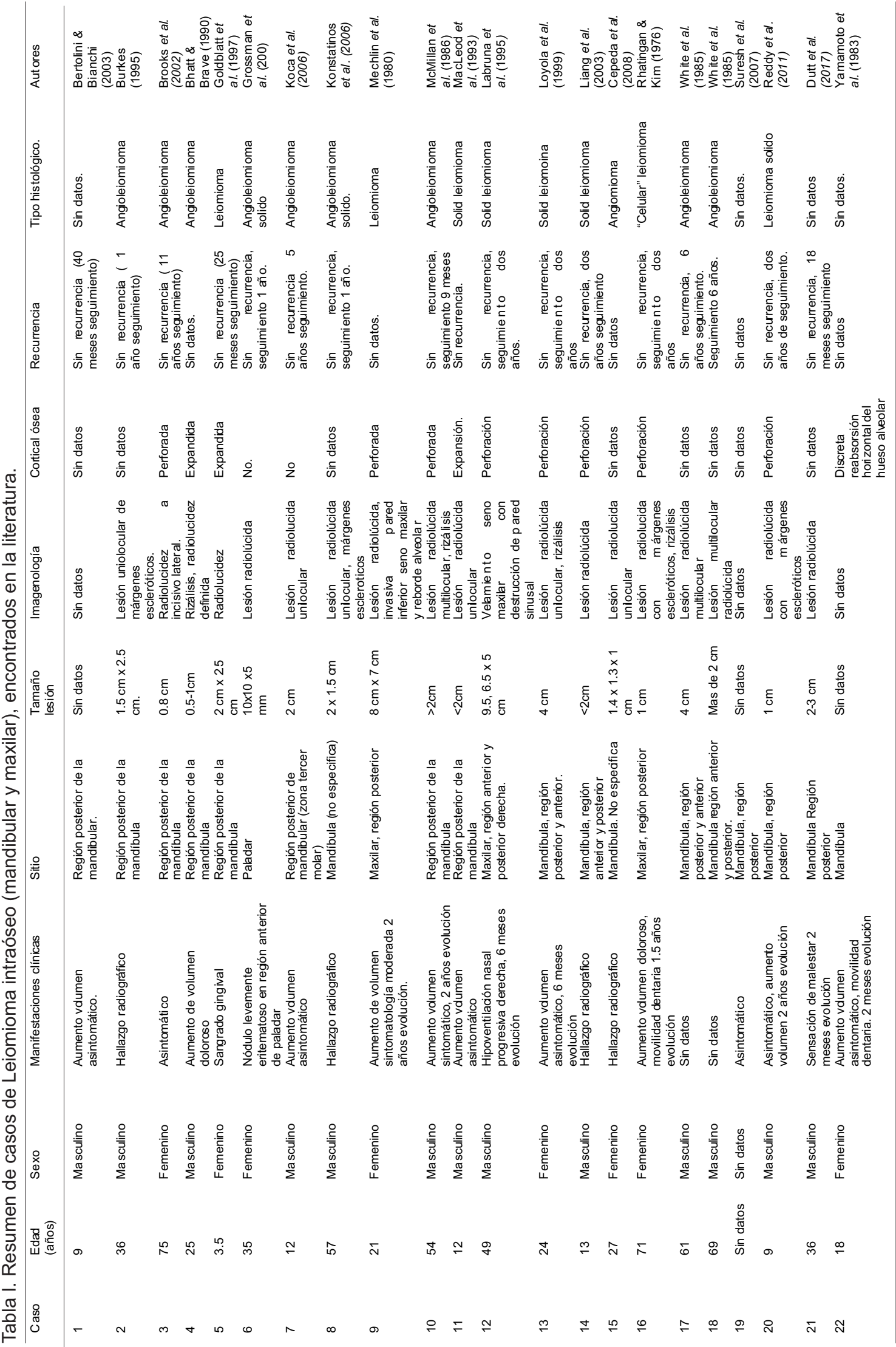



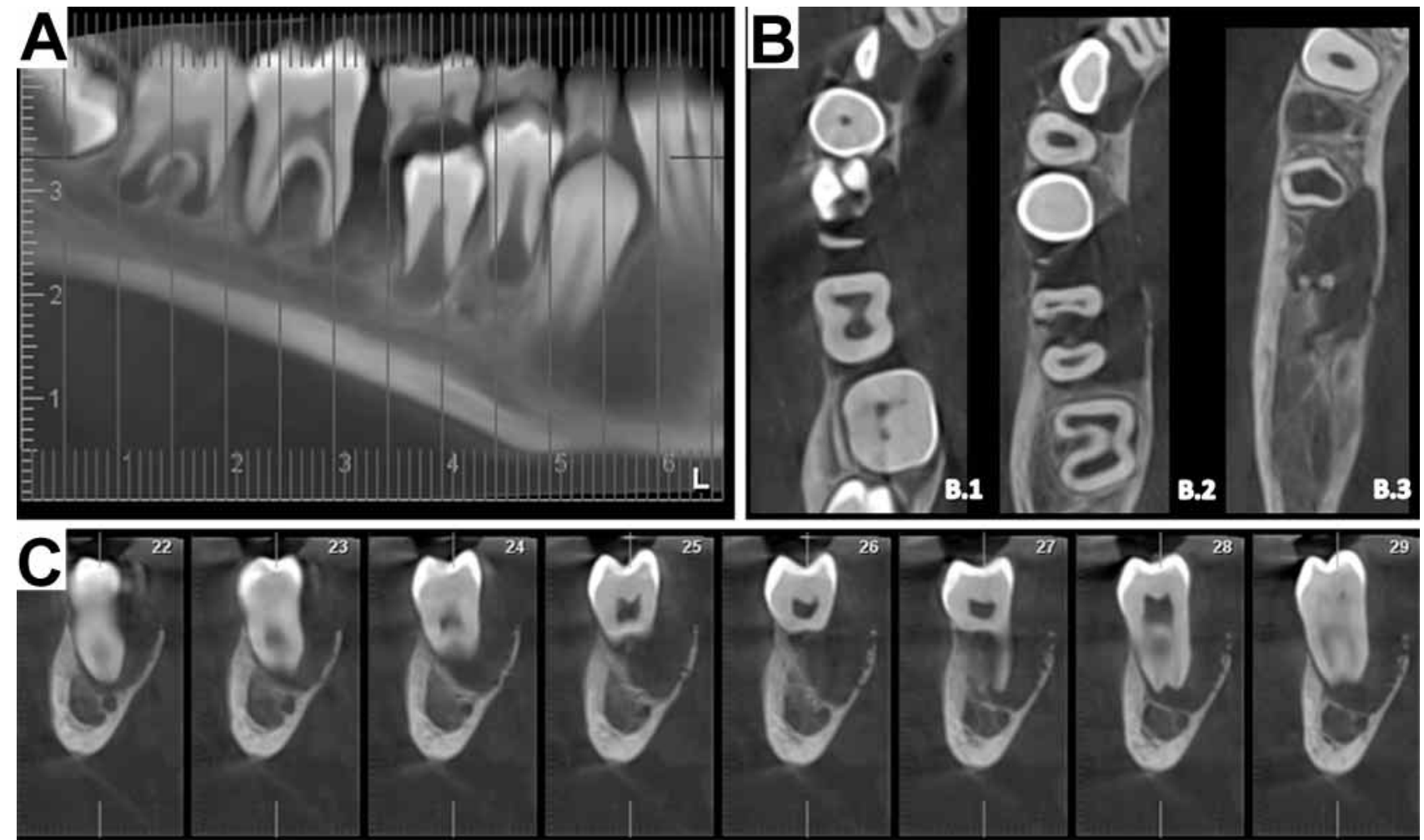

Fig. 1. Imagen Cone Beam de Leiomioma mandibular intraóseo. A. Reconstrucción panorámica de cbct zona mandibular derecha. Cortes en milímetros. Se observa lesion intraósea extendida desde piezas dentarias 19 y temporales K y L. B Cortes axiales. B.1- Tercio superior radicular, B.2- Tercio medio radicular, B.3- Tercio inferior radicular. C. Cortes coronales. Lesión intraósea que aldegaza cortical lingual, sin signos de rizálisis.

ne un estroma de apariencia condroide y puede tener un fenómeno de zonificación, además de ocurrir en pacientes más jóvenes. Otro diagnóstico diferencial es la fascitis nodular, la cual muestra una proliferación de células fusiformes con apariencia de " tissue culture-like ", abundante estroma mixoide o mucinoso, a menudo células gigantes multinucleadas y un infiltrado inflamatorio mixto; los márgenes no están marcadamente demarcados, y existe una positividad citoplásmica para la actina del músculo liso y la actina músculo específica.

En contraste del Leiomioma, tanto el leiomiosarcoma como el fibrosarcoma presentan atipia citológica, y las lesiones de alto grado muestran pleomorfismo y actividad mitótica. Presencia de aumento de celularidad: $\geq 5$ mitosis por 10 campos de mayor aumento y crecimiento infiltrativo (Katou et al., 1997; Loyola et al.).

Otro caso de tumor maligno es el carcinoma sarcomatoide, éste muestra una atipia citológica significativa y positividad para la pancitoqueratina.
Mientras tanto los tumores de vaina de nervio periférico (neurofibroma, schwannoma) muestran reactividad para proteína $\mathrm{S}-100$ y SOX-10.

Diagnóstico. El diagnóstico suele ser difícil por la escasa cantidad de casos en cabeza y cuello y por la semejanza con muchos otros tumores con proliferación de células en forma de huso (fusiformes), como el mucocele, lipoma, neurofibroma, schwanoma, fibroma, fibrosarcoma, miofibro-sarcoma u otras proliferaciones miofibroblásticas (Koca et al., 2006) Por su infrecuencia, radiográficamente el leiomioma intraóseo no se considera dentro de los diagnósticos diferenciales más comunes, sin embargo su aspecto suele dar imágenes radiolúcidas uni o multilocular en la región posterior de la mandíbula, afectando a pacientes de edad joven y media, quienes padecen de reabsorción radicular y cortical, similar a diagnósticos más frecuentes como ameloblastoma, mixoma, quiste óseo traumático, lesión central de células gigantes o sarcomas (Loyola et al.). 
Debe existir cierta precaución a la hora de distinguir un leiomioma de un leiomiosarcoma o un miofibrosarcoma, Koca et al. informaron que el $20 \%$ de los tumores originados en tejido blando en cavidad oral demostraron tener un grado de malignidad, en especial cuando los hallazgos clínicos y radiográficos cuentan con signos sugestivos de malignidad. Diversos autores reportan que en boca la neoplasia se manifiesta con un rápido crecimiento y con mayor agresividad que en otras partes del cuerpo (Katou et al.; Raffaini et al.; McMillan et al.). Leiomiosarcomas tienen una histología que sutilmente puede imitar lesiones benignas. Concordando con Koca et al., consideramos se debe considerar la patología neoplásica maligna en cualquier tumoración de tejido blando hipervascular en la región oral. A diferencia de su contraparte en tejidos blandos, leiomiomas intraóseos han mostrado tendencia a crecer rápidamente. Otros signos, como gran dimensión ( > 30mm) (Loyola et al.), pérdida de láminas corticales, rizálisis, desplazamiento de piezas dentarias. Aún cuando no se logre desmostrar un estado de malignidad de la lesión, si se comprueba un avance significativo, agresividad y otros signos, no debe descartarse una posible lesión maligna, ya que en ocasiones el diagnóstico histopatológico y clínico suele ser complicado.
El Leiomioma mandibular intraóseo se caracteriza por presentar un rápido crecimiento, desplazando y traspasando corticales, con un crecimiento exofítico, invade e infiltra el tejido blando como encía y mucosas adyacentes, su determinación diagnóstica suele ser compleja dada por su baja prevalencia. Debido a su similitud histológica con otro tipos tumoraciones es que no suele ser la primera opción en la lista de diagnósticos diferenciales. Sin embargo es fundamental descartar variantes malignas, como el leiomiosarcoma o miofibrosarcoma. Para esto el análisis de comportamiento clinico, imagenologico y la observación histológica, en ayuda con la microscopía electrónica y marcadores inmunohistoquímicos, suelen ser muy útiles para la pesquisa de esta rara patología neoplásica.

Tratamiento. Los leiomiomas intraóseos mandibulares a diferencia de los leiomiomas extraóseos cuyo tratamiento más frecuente es la exéresis quirúrgica de la lesión bajo anestesia local con una baja o nula recurrencia cuando son tratados de forma correcta, pueden presentar distintos tipos de tratamiento teniendo siempre en cuenta la posibilidad de que estos tumores presentan malignidad.

En particular la variante de Leiomioma intraóseo

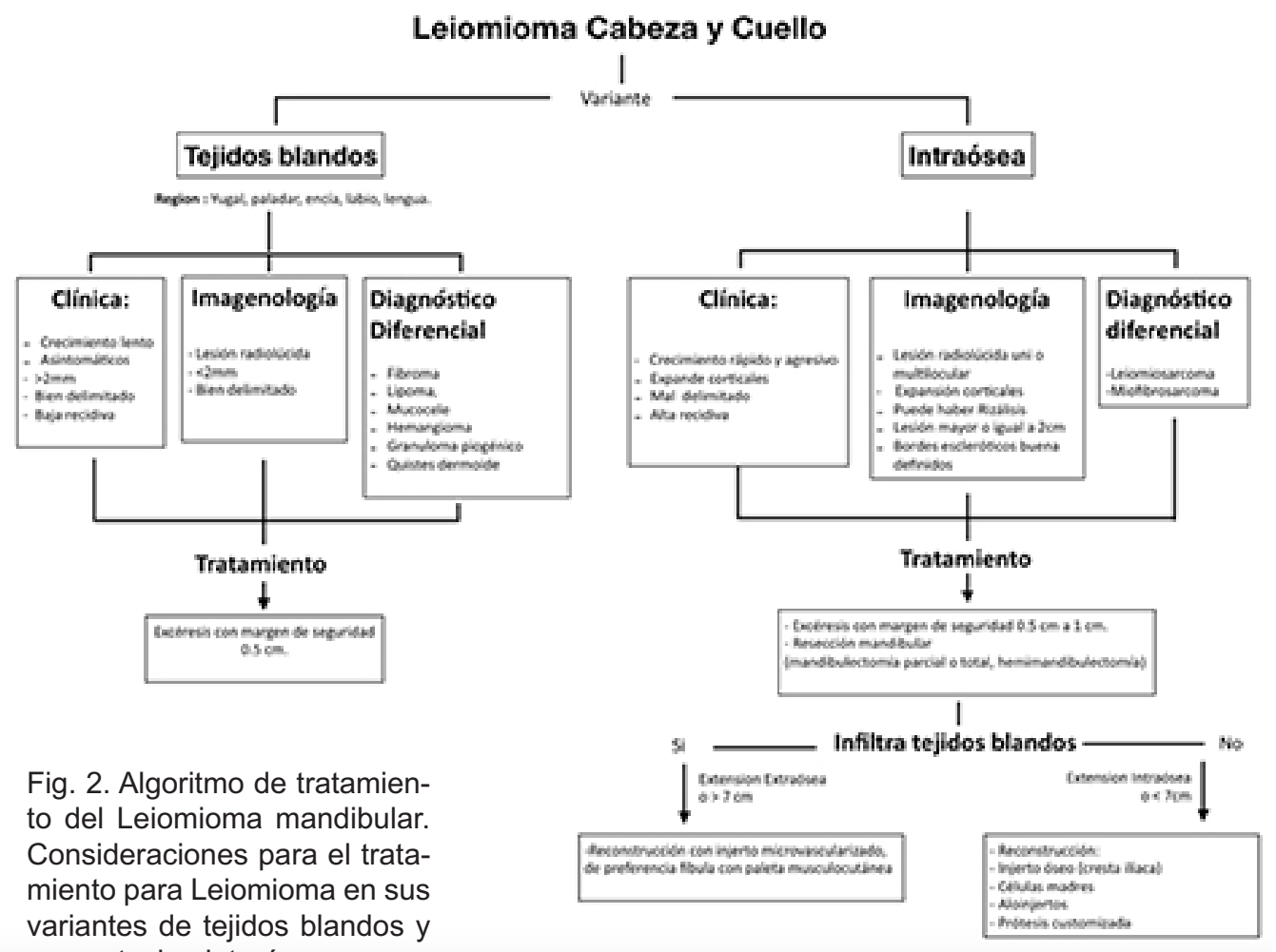
presentacion intraósea. 
mandibular, puede requerir de distintos tratamientos. En general el algoritmo terapéutico va a depender de la extensión de la lesión y si se encuentra tejido blando extraóseo involucrado o no (Fig. 2).

En caso de menores extensiones o que la lesión no infiltre ni invada tejido blando fuera del hueso podemos realizar la resección con margen de seguridad más injertos óseos no vascularizados o aloinjertos, células madres, entre otros. Mientras en el caso de grandes extensiones o infiltración de tejido blando fuera del hueso mandibular, debemos pensar en grandes resecciones, hemimandibulectomía, mandibulectomía total, con márgenes de más de $2 \mathrm{~cm}$ cuando existen dudas de su malignidad, con reconstrucciones basadas en colgajos microvascularizados (Fig. 1).

\section{DISCUSIÓN}

El leiomioma es una tumoración derivada del músculo liso, que afecta principalmente al útero, la piel y sistema gastrointestinal (Eichner). La ocurrencia de esta neoplasia en la región de cabeza y cuello representa un $1 \%$ de los leiomiomas, siendo su ocurrencia en boca infrecuente y su origen en mandíbula aún más raro (Baden et al.). Farman (1975) en un estudio de 7748 casos de neoplasias de músculo liso, encontró que solo 5 se situaron en la cavidad oral. Cuando así ocurre, el leiomioma suele afectar aquellas zonas ricas en tejidos muscular liso proveniente de tejidos como la túnica media de vasos sanguíneos o células mioepiteliales de los conductos excretores de glándulas salivales. De esta forma los sitios más recurrentes suelen ser labios $(27,46$ $\%)$, lengua $(18,30 \%)$, mejillas y paladar $(15,49 \%)$, y encía $(8,45 \%)$. Cuando la ocurrencia es intraósea suele afectar a la mandíbula por sobre el maxilar. Hasta la fecha pocos son los casos de leiomioma intraóseo en la región maxilofacial (Chaudhry et al.). En una revisión electrónica de la literatura realizada por los autores de este artículo, se utilizaron los términos de busqueda (MESH) : "mouth", "oral cavity", "mandibule", "jaw", "Leiomyoma", "Angiomyoma"; ingresandolos en buscadores : PubMed (MEDLINE), Google Scholar y Clinicalkey. Se seleccionaron solo artículos que se encontraran en presentacion full text y en lengua inglesa. Solo se incluyeron aquellos que reportaron claramente casos cuyo origen fue intraóseo (mandíbula o maxilar). Se determinó un total de 12 artículos publicados entre 1986 y 2016 . De 12 articulos identificados en nuestra búsqueda se reportaron 24 casos de leiomiomas en mandíbula (Tabla I). El tipo más frecuente fue el angioleiomioma. El predominio es de hombres (14 casos) sobre mujeres (8 casos), dos articulos no entregaron datos. El rango de edad varió desde 8 meses a 75 años, con un promedio de edad de 35 años. Resultados que concuerdan con los encontrados para las distintas presentaciones de leiomioma en la literatura (Reddy et al., 2011).

Existe una serie de factores que hacen que el Leiomioma intraóseo represente un gran desafío para el cirujano, comenzando con la dificultad del diagnóstico clínico-radiográfico, sumado a la baja recurrencia de éste, necesitando de un equipo multidisciplinario que se componga por el cirujano, patólogo y radiólogo idealmente. Además el diagnóstico diferencial histopatológico es aún controversial entre los clínicos, por lo que requiere de un patólogo experimentado para la diferenciación entre una patología benigna y una maligna. Por otro lado el tratamiento del leiomioma intraóseo a diferencia del leiomioma en tejido blando como encías o mucosas, es de mayor dificultad, requiriendo muchas veces mandibulectomías, hemimandibulectomías o grandes resecciones que involucran tejido blando y tejido óseo, requiriendo por lo tanto reconstrucciones complejas a base de distintas técnicas desde injertos autólogos o aloinjertos, hasta prótesis, reconstrucción con células madre o injertos microvascularizados.

PINO, D. D.; HUENTEQUEO-MOLINA, C.;UNIBAZO, A.; SISO, S. \& OLATE, S. Considerations of Leiomyoma and its intraosseous variant in the jaw, working algorithm according to literature analysis. Int. J. Odontostomat., 14(1):117-123, 2020.

ABSTRACT: Leiomyoma is a benign neoplasm, the origin is the smooth muscle tissue that can be found in any area of the human body, which contains smooth muscle tissue. The most common regions it can be located, are the uterus, the skin and the gastrointestinal tract. The low quantity of muscle tissue in the mouth leads to infrequent oral manifestation, representing only $0.06 \%$ of these tumors. The most affected regions are the lips, cheeks, palate, tongue and gums. The Histologic classification is: Solid Leiomyoma, Angioleiomyoma (vascular) and Epithelioid Leiomyoma (Leiomyoblastoma). The origin of this tumor in the mouth is the tunica media of the blood vessels. Nevertheless, and in spite of being defined as a benign tumor, it can be extremely aggressive, be subject to controversial diagnosis, and must often be treated as a malign neoplasm. The Intraosseous Leiomyoma is infrequent and presents a complicated differential diagnosis, with a histopathology that many times cannot be easily classified. The aim of this article is to present a review of intraosseous variant Leiomyoma in the mandible, the clinicians considerations and a treatment algorithm.

KEY WORDS: oral cavity, mandible, jaw, leiomyoma, angiomyoma, leiomyoma epithelioid. 


\section{REFERENCIAS BIBLIOGRÁFICAS}

Baden, E.; Doyle, J. L. \& Lederman, D. A. Leiomyoma of the oral cavity: a light microscopic and immunohistochemical study with review of the literature from 1884 to 1992. Eur. J. Cancer B. Oral Oncol., 30B(1):1-7, 1994.

Bertolini, F. \& Bianchi, B. Mandibular intraosseous leiomyoma in a child: report of a case. J. Clin. Pediatr. Dent., 27(4):385-7, 2003

Bhatt, A. P. \& Brave, V. R. Angiomyoma of the mandible: a case report. J. Indian Dent. Assoc., 61:298-9, 1990

Brooks, J. K.; Nikitakis, N. G.; Goodman, N. J. \& Levy, B. A. Clinicopathologic characterization of oral angioleiomyomas. Oral Surg. Oral Med. Oral Pathol. Oral Radiol. Endod., 94(2):221-7, 2002.

Burkes, E. J. Jr. Vascular leiomyoma of the mandible: report of a case. J. Oral Maxillofac. Surg., 53:65-6. 1995.

Cepeda, L. A. G.; Rivera, D. Q.; Rocha, F. T.; Huerta, E. R. L. \& Sánchez, E. R. M. Vascular leiomyoma of the oral cavity: Clinical, histopathological and immunohistochemical characteristicspresentation of five cases and review of the literatura Mendez Sánchez. Med. Oral Patol. Oral Cir. Bucal, 13(8):E483-8, 2008.

Dutt, K. C.; Bindra, S.; Awana, M.; Talwar, M. \& Lehl, G. Intraosseous leiomyoma of the mandible: a case report of the rare entity and review of literature. J. Maxillofac. Oral Surg., 16(2):145-51, 2017.

Eichner, E. Adenoid cystic carcinoma of the Bartholin gland. Review of the literature and report of a case. Obstet. Gynecol., 21(5):608-13, 1963.

Farman, A. G. Benign smooth muscle tumours. S. Afr. Med. J., 49(33):1333-40, 1975.

Gaitan Cepeda, L. A.; Quezada Rivera, D.; Tenorio Rocha, F.; Leyva Huerta, E. R. \& Mendez Sánchez, E. R. Vascular leiomyoma of the oral cavity. Clinical, histopathological and immunohistochemical characteristics. Presentation of five cases and review of the literature. Med. Oral Patol. Oral Cir. Bucal, 13(8):E483-8, 2008.

Goldblatt, L. I. \& Edcscss, R. B. Central leiomyoma of the mandible: report of a case with ultrastructural confirmation. Oral Surg. Oral Med. Oral Pathol., 43:591-7, 1997.

Grossmann, S. M.; Johann, A. C.; Castro, W. H.; Friedman, H.; Gomez, R. S. \& Mesquita, R. A. Anterior midline nodule of the hard palate. Oral Surg. Oral Med. Oral Pathol. Oral Radiol. Endod., 108(6):80811, 2009.

Katou, F.; Andoh, N.; Motegi, K. \& Nagura, H. Leiomyoma of the mandible: a rapid growing case with immunohistochemical and electron microscopic observations. Oral Surg. Oral Med. Oral Pathol. Oral Radiol. Endod., 84(1):45-50, 1997.

Koca, H.; Güneri, P.; Çetingül, E. \& Önal, T. A very rare form of leiomyoma: Mandibular angioleiomyoma. Int. J. Pediatr. Otorhinolaryngol. Extra, 1(2):110-4, 2006.

Konstantinos, I.; Melakopoulos, I. \& Patrikiou, A. Intraosseous leiomyoma of the mandible. Oral Oncol. Extra, 42:184-6, 2006.

Labruna A, Reagan B, Papageorge A. Leiomyoma of the maxillary sinus: a diagnostic dilemma. Otolaryngol. Head Neck Surg. 112:595-8, 1995.

Leung, K. W.; Wong, D. Y. \& Li, W. Y. Oral leiomyoma: case report. J. Oral Maxillofac. Surg., 48(7):735-8, 1990.

Liang, H.; Frederiksen, N.; Binnie, W. \& Cheng, Y. Intraosseous oral leiomyoma: systematic review and report of one case. Dentomaxillofac. Radiol., 32:28-90, 2003.

Loyola, A. M.; Araújo, N. S.; Zanetta-Barbosa, D.; Mendes, V. C.; Jordão-Silva, C. \& Bittar, T. O. Intraosseous leiomyoma of the mandible. Oral Surg. Oral Med. Oral Pathol. Oral Radiol. Endod., 87(1):78-82, 1999.

MacLeod, S. P.; Mitchell, D. A. \& Miller, I. D. Intraosseous leiomyoma of the mandible: report of a case. Br. J. Oral Maxillofac. Surg. 31:188, 1993.
McMillan, M. D.; Ferguson, J. W. \& Kardos, T. B. Mandibular vascular leiomyoma. Oral Surg. Oral Med. Oral Pathol., 62(4):427-33, 1986.

Mechlin, D. C.; Hamasaki, C. K.; Moore, J. R. \& Davis, W. E. Templer $\mathrm{J}$. Leiomyoma of the maxilla: report of a case. Laryngoscope, 90:123-3, 1980

Raffaini, M.; Baggi, M. T.; Bozzetti, A.; Sesenna, E. \& Gabrielli, M. Mandibular leiomyoma in an infant. Report of a case. Int. J. Oral Maxillofac. Surg., 19(6):367-9, 1990.

Reddy, B.; Rani, B. S.; Anuradha, Ch.; Chandrasekhar, P.; Shamala, R. \& Lingamaneni, K. Leiomyoma of the mandible in a child. J. Oral Maxillofac. Pathol., 15(1):101-4, 2011.

Rhatingan, R. M. \& Kim, Z. E. Leiomyoma arising adjacent to a maxillary tooth socket: an intraosseous leiomyoma presenting as an odontogenic lesion. South Med. J., 69:493-4, 1976.

Savage, N. W.; Adkins, K. F.; Young, W. G. \& Chapman, P. J. Oral vascular leiomyoma: review of the literature and report of two cases. Aust. Dent. J., 28(6):346-51, 1983.

Schenberg, M. E.; Slootweg, P. J. \& Koole, R. Leiomyosarcomas of the oral cavity. Report of four cases and review of the literature. J. Craniomaxillofac. Surg., 21(8):342-7, 1993.

Suresh, L.; Matdumura, E.; Calixto, L. E.; Ruckert, E. \& Aguirre, A. Intraosseous angiomyoma of the mandible. Gen. Dent., 55(2):132-5. 2007

White, D. K.; Selinger, L. R.; Miller, A. S.; Behr, M. M. \& Damm, D. D. Primary angioleiomyoma of the mandible. J. Oral Maxillofac. Surg., 43(29):640-4, 1985

Yamamoto, H.; Takagi, M.; Otake, S. \& Mori, M. Leiomyoma of the right lower gingiva: a case and the Japanese literature review. J. Oral Maxillofac. Surg., 41:671-5, 1983.

Dirección para correspondencia:

Dr. Claudio Huentequeo Molina

Hospital Dr. Abraham Godoy Peña

Lautaro

CHILE

Email: claudiohuentequeo@gmail.com

Recibido : 03-06-2019

Aceptado: 25-07-2019 